\title{
The Effect of HIV Related Risk Factors on HIV Status: A Case of Gamo-Gofa Free Standing Voluntary Counseling and Testing Center
}

\author{
Tesfahun Zewde Legisso, Markos Abiso Erango \\ Department of Statistics, Arba Minch University, Arba Minch, Ethiopia \\ Email address: \\ tesfahunzewde@yahoo.com (T. Z. Legisso), markos.erango73@gmail.com (M. A. Erango) \\ To cite this article: \\ Tesfahun Zewde Legisso, Markos Abiso Erango. The Effect of HIV Related Risk Factors on HIV Status: A Case of Gamo-Gofa Free \\ Standing Voluntary Counseling and Testing Center. Science Journal of Applied Mathematics and Statistics. Vol. 6, No. 4, 2018 , pp. $130-134$. \\ doi: $10.11648 /$ j.sjams.20180604.14
}

Received: July 28, 2018; Accepted: October 17, 2018; Published: November 12, 2018

\begin{abstract}
HIV/AIDS is a major development concern in many countries and is destroying the lives and livelihoods of many people around the world. This study is aimed to assess the demographic and HIV related risk behavior factors that may affect HIV status of the visitors of VCT centers. A cross sectional study was conducted in Gamo-Gofa districts, Southern Nations Nationalities and Peoples Regional State of Ethiopia. A total of 4028 sample were selected using stratified random sampling technique. Data were collected with a designed questionnaire from 20 voluntary counseling and testing center of the districts. If the clients visit VCT center is HIV-infected, it is categorized as HIV positive and if the client test is indicated not HIVinfected, then the visitor categorized as HIV negative status. The Binary logistic regression model was used to analyze the data using the SPSS software. The results of the study revealed that the probability of an individual being HIV positive was 0.0286 and the predictor's variables age, marriage status, education level, alcohol use, knowledge about HIV, monthly income, condom use and residence of the individual were significantly effect on being HIV-positive. Health professionals and responsible bodies should work on these significant variables to reduce the probability of being HIV positive.
\end{abstract}

Keywords: HIV, Logistic Regression, VCT

\section{Introduction}

\subsection{Background}

Acquired Immune Deficiency Syndrome (AIDS) which is believed to be caused by the Human Immunodeficiency Virus (HIV) has been the major problem worldwide. The rate of spread of the HIV/AIDS epidemic is still high. The expansion of the epidemic has become a burning issue globally and this is particularly so more in developing countries [1].

Globally around 34 million people were living with HIV; 2.7 million new infections occurred and 1.8 million people [1.6 million-1.9 million] died from AIDS-related causes in 2010. The burden of the epidemic vary considerably between countries and regions, the Sub-Saharan Africa remains the most severely affected region accounting for nearly $68 \%$ of the people living with HIV worldwide. In Sub-Saharan
Africa around 22.9million people were living with HIV; 1.9millon new infection occurred and 1.2 million people died from AIDS related causes [2].

The socioeconomic consequences of the pandemic, however, have proved difficult to assess. Economic impacts are probably easier to define (poverty, income, wealth/assets/savings), whereas social impacts can range widely from physical and mental health to schooling and social networks (including social stigma) to demographic outcomes such as marriage, fertility and migration. There are many routes through which AIDS could affect socioeconomic outcomes. Obviously AIDS-related morbidity and mortality affect the health and life expectancy of an infected person, and these effects can spread to the caregivers or survivors in the household, the extended family, the community and the nation. Families incur direct costs of illness and death (medical expenditures, funeral costs) and indirect costs (loss of labor and income) as well as declines 
in psychosocial well-being [3].

The effect of HIV/AIDS takes several forms, at the household, community and national levels. It stands out among contemporary fatal infectious human diseases and pervades all socio-economic classes. HIV/AIDS affects households, children, and community in several ways. According to Franklin Simtowe and et al, HIV/AIDS affected families suffer loss of productive labor, loss of income, loss of food reserves, savings and assets which are diverted to meet health care and funeral costs [4].

The Demographic and Health Survey data from developing countries specially sub-Saharan suggest that wealthier men and women tend to have higher prevalence of HIV than the poorer ones [5]. On the other hand, other studies showed that poverty increases individual exposure to HIV infection and it is a key factor for HIV transmission.

\subsection{Definitions and Concepts of VCT}

VCT is recognized as one of a few potential effective and affordable methods for reducing the transmission of HIV in developing country. CT program has been identified as an entry point for and care and support services. Different interventions shows that counseling and testing based on knowledge of their anti-body status; enables individuals to make informed choices; helps them to change behaviors that may put themselves and others at risk for HIV infection, take action to prevent prenatal transmission once person learn they are infected, and also to seek early medical care if they are HIV positive. HIV is a worldwide infection and very challenging disease. Approximately 39.5 million people worldwide were living with HIV in 2006 [1].

HIV counseling has been defined as: "A confidential dialogue between a person and a care provider aimed at enabling the person to cope with stress and make personal decisions related to HIV/AIDS. The counseling process includes an evaluation of personal risk of HIV transmission and facilitation of preventive behavior. The purpose of HIV counseling are the prevention of HIV transmission and the emotional support of those who wish to consider HIV testing, both to help them make a decision about whether or not to be tested, and to provide support and facilitated decision making following testing. People often come for HIV testing in states of considerable anxiety for their health, HIV counseling can be carried out anywhere that provides an environment that ensures confidentiality and allows for private discussion of sexual matters and personal worries. Counseling must be flexible and focused on the individual client's specific needs and situation. In some settings HIV counseling is available without testing. This may help also promote changes in sexual risk behavior.

HIV testing may have far-reaching implications and consequences for the person being tested. Although there are important benefits to knowing one's HIV status, HIV is, in many communities, a stigmatizing condition, and this can lead to negative outcomes for some people following testing. These include ostracism, loss of livelihood, and of family and community respect [6].
Confidentiality: Many people are afraid to seek HIV services because they fear stigma and discrimination from their families and community, and loss of employment. VCT services should therefore always preserve individuals' needs for confidentiality. Trust between the counselor and client enhances adherence to care, and discussion of HIV prevention.

\section{Methods}

The study population consisted of 94840 clients who visited the Gamo Gofa districts free standing VCT centres in study period 2012 to 2013. A cross-sectional survey was conducted with a stratified random sampling technique on 4028 samples from the total population. The sample size (4028) was determined with appropriate derivations. The response variable was an aggregate measure of the HIV status of clients. It is dichotomized as HIV positive (coded as 1) if his/her test is as negative status (coded as 0 ). The explanatory variables included in this study were sex, age, marital status, educational level, employment status, why client is here today, previously tested, ever had sex with penetration, Confidence of the individual, knowledge about HIV, economical status, marital status, condom use, number of sexual partners, Did you checked before, With how many people have you made sex, From whom have you met, Do you tell, Do you have Knowledge about HIV, do you drink alcohol and when do you spend most of the time and other related variable are the potential risk factors considered in this study.

\section{Logistic Regression Model}

The response variable is HIV status of an individual, denoted by $\mathrm{Y}$, which is dichotomous with outcome probability either positive status with probability $\mathrm{P}_{\mathrm{i}}=$ $\mathrm{P}\left[\mathrm{Y}_{\mathrm{i}}=1 \mid \mathrm{X}_{\mathrm{i}}\right.$ or with negative status $\mathrm{P}_{\mathrm{i}}=\mathrm{P}\left[\mathrm{Y}_{\mathrm{i}}=0 \mid \mathrm{X}_{\mathrm{i}}\right.$, where $X_{i}$ is a vector of $r$ factors or predators for each individual $\mathrm{i}=1,2,3 \cdots \mathrm{n}$

The logistic regression model is given as [7]

$$
p_{i}=\frac{e^{\beta_{0}+\beta_{1} x_{i 1}+\beta_{2} x_{i 2}+\cdots \beta_{r} x_{i r}}}{1+e^{\beta_{0}+\beta_{1} x_{i 1}+\beta_{2} x_{i 2}+\cdots \beta_{r} x_{i r}}}
$$

and equivalently expressed with the logit link function as

$$
\operatorname{logit}\left(p_{i}\right)=\beta_{0}+\beta_{1} x_{i 1}+\beta_{2} x_{i 2}+\cdots \beta_{r} x_{i r}
$$

Where $i=1,2,3 \cdots n, j=0,1,2, \cdots r, X_{i j}$ the $j$ th predictor of the ith an individual is, $\beta_{0}$ is an intercept, $\beta_{\mathrm{i}} \mathrm{s}$ are coefficients of the predictors variables.

\subsection{Estimation of the Parameters}

The likelihood function $\mathrm{L}(\mathrm{y} \mid \mathrm{X}, \beta)$ is defined as the joint probability distribution $f(y \mid X, \beta)$ of the independent observation vector of size $n$ given the regression parameters $\beta$ and the design matrix $X$. The likelihood function with the $n$ independent observations is expressed as 


$$
L(y \mid X, \beta)=\left(\frac{e^{\beta_{0}+\beta_{1} x_{i 1}+\beta_{2} x_{i 2}+\cdots \beta_{r} x_{i r}}}{1+e^{\beta_{0}+\beta_{1} x_{i 1}+\beta_{2} x_{i 2}+\cdots \beta_{r} x_{i r}}}\right)^{y_{i}}\left(\frac{1}{1+e^{\beta_{0}+\beta_{1} x_{i 1}+\beta_{2} x_{i 2}+\cdots \beta_{r} x_{i r}}}\right)^{1-y_{i}}
$$

It refers to how likely a particular population is to produce an observed sample given the parameter values. The logistic regression model is nonlinear so that Newton-Raphson iterative algorithm is necessary for parameter estimation. The general method of estimation that leads to the least squares function under the linear regression is maximum likelihood estimation. It is this method that provides the foundation for the approach to estimation with the logistic regression model. In a very general sense the method of maximum likelihood yields values for the unknown parameters which maximize the probability of obtaining the observed set of data.

\subsection{Assessing the Fit of the Model}

Measures of goodness of fit are statistical tools used to explore the extent to which the fitted response obtained from the postulated model compares with the observed data. Clearly, the fit is good if there is a good agreement between the fitted and the observed data. There is no guarantee, however, that a particular model of this form is appropriate or that it provides a good fit to the data. So, the study needs to assess the goodness of fit of the model [7].

The Hosmer-Lemeshow test is a commonly used test for assessing the goodness of fit of a model and allows for any number of explanatory variables, which may be continuous or categorical. This measures the correspondence between the actual and predicted values of the dependent variable. The test is similar to that of chi-square goodness of fit test and has the advantage of partitioning the observations into groups of approximately equal size, and therefore there are less likely to be groups with very low observed and expected frequencies. In this case, better model fit is indicated by a smaller difference in the observed and predicted classification [8].

\section{Result and Discussions}

Among the 4,028 samples considered, 64\% were females and $36 \%$ were males. Approximately $41 \%$ and $46 \%$ of the respondents in age category of 18-30 and 31-40 respectively and the rest of the respondent's age were more than 41 . Among considered samples, approximately 41\%, 51\% and $8 \%$ of the respondents were single, married and Divorced/widowed marital status respectively and regarding education level $11 \%, 24 \%, 30 \%$ and $35 \%$ were illiterate, primary, secondary school and certificate and above respectively. Among considered sample, approximately $22 \%$ were farmer, $2 \%$ were commercial sex workers, $21 \%$ were daily workers, $33 \%$ were Trader/driver and 225 were civil servants. Regarding knowledge about HIV, among considered sample approximately 3\% have no knowledge about HIV, 47\% have little knowledge and 50\% have knowledge about HIV. Among considered samples 34\% were drink alcohol and 56\% were not drink alcohols. In relation with the residence of the respondents $70 \%$ of the respondents were live in urban area and $30 \%$ were live in rural area. The analysis shows that $2.86 \%$ of the respondents were HIV positive status and $97.14 \%$ were HIV negative status.

Tests to find out the association between the individual HIV status and the factors were carried out using the chisquare tests. Most of the factors were significantly associated with the HIV status of individual at the significant level of $5 \%$. The results displayed in Table 1 showed that the fifteen variables like sex, age, marital status, education level, alcohol use, condom use, spend time, Knowledge about HIV, number of sex partners, income, why are you here today, did you checked before, have you ever had sex, with whom you made sex and why you do not want to tell were significantly correlated with the HIV status.

Table 1. Test of association of predictor variable on HIV status.

\begin{tabular}{llll}
\hline \multirow{2}{*}{ Predictor Variable } & HIV status & \\
\cline { 2 - 4 } & Chi-square & df & P-value \\
\hline Sex & 61.877 & 1 & 0.00 \\
Age & 19.747 & 2 & 0.000 \\
Marital status & 224.6 & 3 & 0.000 \\
Education & 25.14 & 2 & 0.000 \\
Residence & 0.06 & 1 & 0.006 \\
Alcohol use & 31.29 & 1 & 0.000 \\
Without condom & 44.95 & 1 & 0.000 \\
Income & 92.3 & 2 & 0.000 \\
Spend time & 5.48 & 3 & 0.043 \\
Knowledge & 19.20 & 2 & 0.000 \\
Religious & 4.41 & 3 & 0.061 \\
No of sex partner & 80.05 & 2 & 0.000 \\
Occupation/employment & 12.47 & 3 & 0.054 \\
Why are you here today & 7.26 & 2 & 0.045 \\
Did you checked before & 16.23 & 1 & 0.050 \\
Have you ever had sex & 59.65 & 1 & 0.000 \\
with whom have you made sex & 4.47 & 3 & 0.050 \\
Do you want to tell the result & 1.97 & 1 & 0.164 \\
Why you do not want to tell & 1.63 & 2 & 0.045 \\
Do you carry about others & 5.86 & 1 & 0.056 \\
Abbreviation: df, degrees of freedom & & & \\
\hline
\end{tabular}

To check the goodness-of-fit of the model, the HosmerLemeshow test was used. The chi-square test statistic value was $(\chi 2=13.859)$, with 8 degrees of freedom at the significant level of 0.186 . Hence, there was not enough evidence to reject the null hypothesis that the model fits the data. Moreover, the likelihood ratio test (deviance) was applied to test the difference between the null and the final model, with the results of -2 Log likelihood to be 840.70 for the null model and 245.43 for the final model. The likelihood ratio test is 595.27 with a 10 degrees of freedom.

Results of the logistic regression analysis are displayed in Table 2. The factors such as sex, age, marriage status, education level, alcohols use, and residence of the individual, condom use, income and knowledge about HIV had significant effect on HIV status of the individual.

The odds ratios can be interpreted as follows: 
The odd of being infected with HIV was 5.735 times for individual whose gender status is female compared to that whose gender status is Male. This indicated that female were more likely to be HIV infected with compare to men. The odds of being infected with HIV was 4.265 times for the individual with age groups from 18 to 30 and 1.318 times for individual with age group from $31-40$ as compare to individual with age groups more than 40, other factors controlled. This indicates that the respondent in age group from 18 to 30 and 30 to 40 were more exposed to HIV infection as compare to age groups more than 40 . So, being in this group ages are induces more risk of being HIV infected than other age groups.

Table 2. Analysis of Logistic Regression Model

\begin{tabular}{|c|c|c|c|c|c|c|c|}
\hline Predictor & Category & $\widehat{\boldsymbol{\beta}}$ & S. $\mathbf{E}(\widehat{\boldsymbol{\beta}})$ & Wald & df & Sig & $\operatorname{Exp}(\widehat{\beta})$ \\
\hline Constant & & 15.16 & 9.05 & 2.81 & 1 & 0.094 & 3.856 \\
\hline \multirow[t]{2}{*}{ Sex } & Female & 1.747 & 0.56 & 9.78 & 1 & 0.002 & 5.736 \\
\hline & $>40$ & - & - & 15.68 & 2 & 0.000 & \\
\hline \multirow[t]{3}{*}{ Age } & $30-40$ & 1.15 & 0.56 & 2.41 & 1 & 0.021 & 1.318 \\
\hline & $18-30$ & 2.658 & 0.41 & 8.61 & 1 & 0.003 & 4.265 \\
\hline & Single & & & 14.63 & 2 & 0.002 & \\
\hline \multirow[t]{2}{*}{ Marital status } & Married & 2.48 & 0.96 & 6.15 & 1 & 0.046 & 1.031 \\
\hline & illiterate & & & 9.23 & 3 & 0.026 & \\
\hline \multirow{3}{*}{ Education Level } & Elementary & 0.62 & 0.435 & 3.96 & 1 & 0.037 & 1.862 \\
\hline & High school & 1.32 & 0.28 & 7.43 & 1 & 0.006 & 3.725 \\
\hline & Certificate $\&$ above & 0.861 & 0.36 & 5.36 & 1 & 0.025 & 2.365 \\
\hline Residence & Rural area & -2.33 & 0.63 & 13.53 & 1 & 0.000 & 0.097 \\
\hline Alcohols use & users & 1.67 & 0.54 & 8.68 & 1 & 0.030 & 2.540 \\
\hline Having Sex & Without condom use & 4.326 & 0.34 & 62.68 & 1 & 0.000 & 5.669 \\
\hline \multirow{2}{*}{ Monthly Income } & Above 1000 & 5.43 & 0.179 & 8.17 & 1 & 0.000 & 4.040 \\
\hline & Nothing & & & 31.42 & 2 & 0.000 & \\
\hline \multirow[t]{2}{*}{ Knowledge about HIV } & Little & -4.015 & 1.553 & 6.681 & 1 & 0.010 & 0.018 \\
\hline & A lot & 1.801 & 0.421 & 18.304 & 1 & 0.000 & 2.057 \\
\hline
\end{tabular}

The odd of being infected with HIV was 1.862 times for the person with elementary education level, 3.725 times for the persons High school and 2.365 times for the person with education level Certificate $\&$ above as compare to the person not education. The odd of being infected with HIV was decreased by $90.3 \%$ for a person who lives in rural area. This indicate that peoples live in urban area is more likely infected with HIV than peoples live in rural area.

The likelihood of being HIV positive is 2.54 times higher for alcohol users than not alcohol users. The person who had sex without condom use was 5.67 times more infected than the person who used condom. When come to the economic background the individuals with income less than 500 and between 500 and 1000 were 4.04 and 1.051 times more infected by HIV/AIDS than the individuals whose income above 1000 birr. The likelihood of being HIV/AIDS positive is 2.057 times higher for individual with no knowledge about HIV than those who have more knowledge. In relation to marital status, the odd of being infected with HIV was 1.031 times for married, 3.033times for divorced/widowed person as compared to single. This indicated that the person whose marital status was married and divorced were more exposed for HIV infection.

The study has provided an insight in to the factors that determine the infection of HIV/AIDS in Gamo Gofa districts. To identify the main determinant factors that affect HIV status in the districts, the primary data were collected from 4028 visitors that visit the VCT center using questioners during the study period. One of the key demographic characteristics was sex. Female was more likely to be HIV infected compared with men. Studies revealed that HIV infection was higher in girls than boys. This is due to the fact that at young age, boys have sex with girls of similar age, whereas girls have sexual relations with older men, who are more likely to be infected [9]. Regarding age of the respondent, age groups 18-30 more exposed for HIV infection compared to other age groups. According to Da Ros and Da Silva Schmitt (2008) sixty percent of new infection affects the young age group globally [10].

According to the Ethiopian Demography and Health Survey (EDHS), 2011, reports the prevalence rate was significantly higher among divorced/separated and widowed individuals as compared to married and single individuals [11]. This study also showed that HIV prevalence was significantly higher among divorced or separated and singe individuals compared to married individual. Education is the other socio-economic factor, which influences the prevalence of HIV. This study showed that HIV prevalence increases significantly with increment of education level. According to Hargreaves JR (2010) also higher HIV prevalence is observed among more educated categories [9]. But a study conducted by (Bradley, et al., 2007), showed that HIV prevalence decreases significantly with each increase in education level [12].

This study tells us that the prevalence rate is higher in urban areas than rural areas. According to Mmbaga, et al(2007) the prevalence of HIV infection in urban, semiurban and rural areas in Arusha region, Tanzania showed that 
the prevalence rate is higher in urban areas than rural areas [5]. In this study the likelihood of being HIV positive is higher for alcohol users than not alcohol users.

\section{Conclusion}

The aim of this study was to assess the demographic and HIV related risk behavior factors that may affect HIV status of the visitors of VCT centers. The analysis revealed that $2.86 \%$ individuals were infected by HIV. From the logistic regression model analysis, nine risk factors were found to be significant at $5 \%$ significance level. Increased odds of risk to being HIV infected was due to sex, age, education level, marital status, residence of the individual, Alcohols use, Sex Without, monthly income and knowledge about HIV. In the study areas the youngest peoples were more exposed for HIV infection compared to the oldest people.

In conclusion, a significant number of individual are found to be HIV infected, and so they need greater attention and appropriate intervention.

\section{Acknowledgements}

The authors would like to thank the anonymous reviewers for their comments that were useful to improve the write-up of the paper and Arba Minch University research office for financial support.

\section{References}

[1] UNAIDS/WHO.2010. AIDS Epidemic Update. Washington D. C., USA: USAID.
[2] UNAIDS 2011 World AIDS Day report.

[3] Beegle, K., De Weerdt, J., Dercon, S. (2008). “Adult mortality and consumption growth in the age of HIV/AIDS.

[4] Franklin Simtowe, and Kinkingninhoun-Medagbe F. M (2011): The impact of HIV/AIDS on labor markets, productivity and welfare in Southern Africa, African Journal of Agricultural Research Vol. 6(10), pp. 2118-2131.

[5] Mmbaga, E. J., G. H. Leyna, K. S. Mnyika, A. Hussain, and K. I. Klepp. 2007c. "Education Attainment and the Risk of HIV-1 Infections in Rural Kilimanjaro Region of Tanzania.

[6] World Health Organization. HIV/AIDS Testing and Counseling. http://www. who. int/hiv/topics/vct/en. Accessed March5, 2012. Kilimanjaro region of Tanzania: Implications for prevention and treatment.

[7] Agresti A. An Introduction to Categorical Data Analysis. 3rd ed. New York: John Wiley and Sons Inc.; 1996.

[8] Bewick et al., (2005). Introduction. Logistic regression provides a method for modelling a binary response variable.

[9] Hargreaves JR, H. L., 2010. Changes in HIV prevalence among differently educated groups in Tanzania between 2003 and 2007.

[10] Da Ros, C. \& Da Silva Schmitt, C., 2008. Global epidemiology of sexually transmitted diseases, Asian Journal of Andrology. Asian Journal of Andrology, 10(1), pp. 110114.

[11] Central Statistical Agency, 2011. Ethiopia Demographic and Health Survey, Addis Ababa: USAID.

[12] Bradley, H. et al., 2007. Educational Attainment and HIV Status among Ethiopian Voluntary Counseling and Testing Clients. AIDS and Behavior, 11(5), pp. 736-742. g. 